\title{
Potential of 3D Surface Imaging for Quantitative Analysis of Fat Grafting
}

\author{
Audrey L. CHEONG ${ }^{1}$, Summer E. HANSON ${ }^{2}$, Gregory P. REECE ${ }^{2}$, \\ Mia K. MARKEY ${ }^{3,4}$, Fatima MERCHANT ${ }^{\star 1,5}$ \\ ${ }^{1}$ Department of Electrical and Computer Engineering, University of Houston, USA; \\ ${ }^{2}$ Department of Plastic Surgery, The University of Texas MD Anderson Cancer Center, Houston, USA; \\ ${ }^{3}$ Department of Biomedical Engineering, The University of Texas at Austin, USA; \\ ${ }^{4}$ Department of Imaging Physics, The University of Texas MD Anderson Cancer Center, Houston, USA; \\ ${ }^{5}$ Department of Engineering Technology, University of Houston, USA
}

DOI: $10.15221 / 18.057 \quad$ http://dx.doi.org/10.15221/18.057

\begin{abstract}
Autologous fat grafting is increasingly employed to address volume asymmetry and contour irregularity following breast reconstruction for breast cancer. However, there are no well-established objective tools to accurately measure change in graft volume and breast shape over time. Three-dimensional (3D) surface imaging allows for objective analysis of changes in breast shape and size, which clinicians and researchers can use to evaluate the effects of fat grafting. This study presents several measurements (Gaussian curvature, shape index, surface orientation, and volume) that can be extracted from 3D surface images of patients. These measurements are demonstrated on three patients (two patients after implant reconstruction and one after bilateral mastopexy) before and after fat grafting. The results of this study can help pave the way for clinicians and researchers to develop standardized metrics for objectively evaluating fat graft processing techniques. Using the proposed measurements, we were able to evaluate key shape and size differences in the 3D surface images before and after fat grafting.
\end{abstract}

Keywords: 3D surface image, fat grafting, shape analysis, quantitative analysis

\section{Introduction}

Autologous fat grafting is increasingly employed to address volume asymmetry and contour irregularity following breast reconstruction for breast cancer. One challenge clinicians face is the highly variable reported rates of graft resorption. Clinical outcomes studies are limited in patient volume, retrospective design, and poorly defined outcome measures. Furthermore, there are no well-established objective tools to accurately measure change in graft/breast volume and breast shape over time.

Previous studies have qualitatively or semi-quantitatively assessed various fat grafting techniques to determine which technique had the best outcomes and the highest retention rates [1]-[3]. Other factors, such as radiation therapy and reconstruction procedures, affecting volume retention rates have also been studied [4]. Many studies have used unique measures, such as the fat graft weight [1] and volume [4], [5], soft tissue thickness [3], number of complications and number of injections [6], and cellular integrity [2]. Surgeons and patients may also be given a custom evaluation scale to rate outcomes, such as pigmentation, vascularization, pliability, thickness, itching, pain, and overall satisfaction [2], [7]. In this study, we propose objective metrics to evaluate breast shape and volume over time.

\section{Study Sample}

Three consented breast cancer patients, who already underwent their primary breast reconstruction procedure, were imaged before and after fat grafting at The University of Texas MD Anderson Cancer Center. Three-dimensional surface torso images were captured using a stereo-photogrammetric imaging system (3dMDTorso, 3dMD Inc., Atlanta, GA). During imaging, the cameras were at a fixed distance from the subjects, and all subjects were standing upright with their arms stretched out to the sides to avoid camera obstruction. Demographic details of the three subjects are shown in Table 1. Patients $A$ and $B$ had implants that were placed subpectorally (Fig. 1 and Fig. 2). Patient $C$ had an earlier mastopexy for cosmetic reasons, and later underwent a lumpectomy (Fig. 3).

\footnotetext{
* fmerchant@uh.edu
} 
Table 1. Patient demographics and surgical procedure.

\begin{tabular}{|c|c|c|c|c|c|}
\hline Patient & Age & BMI & Race/Ethnicity & Surgery & Total Fat Graft Volume \\
\hline $\mathrm{A}$ & 52 & 26.4 & Caucasian/NH & $\begin{array}{c}\text { Implant-based } \\
\text { reconstruction }\end{array}$ & $100 \mathrm{cc}$ \\
\hline $\mathrm{B}$ & 27 & 27.4 & Caucasian/NH & $\begin{array}{l}\text { Implant-based } \\
\text { reconstruction }\end{array}$ & $120 \mathrm{cc}$ \\
\hline $\mathrm{C}$ & 61 & 23.8 & Caucasian/NH & $\begin{array}{c}\text { Cosmetic Mastopexy } \\
\text { and then Lumpectomy }\end{array}$ & $360 \mathrm{cc}$ \\
\hline
\end{tabular}

NH Not Hispanic

\section{Methodology}

\subsection{Image Cropping and Alignment}

Each 3D surface image was manually aligned to the front forward facing pose such that the torso height was along the $y$ axis, width was along the $x$ axis, and the breasts projected along the positive $z$ axis. Then the images were manually cropped, removing the arms, neck, and lower torso, using software developed in our lab to visualize and perform measurements on 3D images of the torso. Next, the pre- and post-fat grafting images for each patient were manually registered by visually aligning the sternal notch, the lateral sides of the torso, the midline, and the inframammary fold (if visible) through translation and rotation of the post-graft image with the pre-graft image as the reference.

\subsection{D Measurements}

Following alignment, Gaussian curvature, the shape index, and the surface orientation [8] were computed for each vertex in the cropped images. To evaluate any changes in breast shape occurring due to the fat grafting procedure, difference images were created based on the computed values of curvature, shape index and surface orientation. To compute differences, each reference vertex in the pre-image was evaluated against the closest vertex (as determined by the Euclidean distance), in the aligned post-image(s). The difference image was normalized such that intensity values from 0 to 255 represent changes, with no change shown in black (0), and maximal change shown in white (255).

Gaussian curvature was computed using a toolbox developed by Peyre [9] based on algorithms proposed by Cohen-Steiner [10], [11]. A 10-ring neighborhood, as suggested by the author, was used to calculate the curvature tensor. The surface orientation is defined for each vertex as the angle between the outward surface normal vector of the vertex on the surface mesh and a forward-pointing vector (positive $z$ direction). These angles can range from 0 to 180 degrees.

\subsection{Demarcation of the Fat Graft Region of Interest (ROI)}

The fat grafted regions were manually demarcated on the post-graft images for the three patients by a plastic surgeon. The outlines were then superimposed on both the pre- and post-graft images to show changes specific to the fat grafting region. Boundary points were manually defined on the images following the outlines, and Dijkstra's shortest path algorithm was used to connect the boundary points. The vertices within the defined boundary points were labeled as the fat graft area.

\subsection{Breast Volume Computation}

For volume computation, four points, enclosing the entire breast, were manually located for each breast at the anterior axillary point, lateral point, a point along the midline above the breasts, and a point along the midline near the medial side of the inframammary fold. Volume was computed using the method described in [12], which uses a generated Coons patch to represent the chest wall, and the space between the breast mesh and the Coons patch is measured as the volume.

\section{Results and Discussion}

A previous study by Losken et al. [6] noted that, in the majority of cases, fat was injected to the upper pole of the breast in implant reconstructed patients in order to provide a more natural transition between the upper chest wall and the implant. The second most common location of fat injection was the medial quadrant. The patients presented in this study also had fat injected mainly in the upper and medial quadrants of the breast. One patient had fat injected in the lateral quadrant of the breast.

The volume of the entire breast mound was computed but was not found to show large differences for Patients A and B (Fig. 1 and Fig. 2). However, Patient C, who received the highest fat injection volume, showed a large volume difference (Fig. 3). Assuming volume changes were due to the injected fat alone, fat volume retention was below $25 \%$ for all three patients. Breast volume in Patient $A$ increased 
by $15 \mathrm{cc}$ in the left breast one month after $100 \mathrm{cc}$ of fat was injected, indicating a $15 \%$ retention rate after 1 month, and then decreased by $2 \mathrm{cc}$ after another 5 months (13\% retention rate). For Patient B, 19 days after $60 \mathrm{~mL}$ of fat was injected to each breast, a $21 \mathrm{cc}$ volume increase in the right breast and a 19 cc volume decrease in the left breast were observed. Interestingly, the volume measured before the fat injections and 3 months after the fat injections were the same for Patient $B$, suggesting zero fat retention. Patient $\mathrm{C}$ had the most notable change in volume with an $80 \mathrm{cc}$ volume retention out of the $360 \mathrm{cc}$ $(22.2 \%)$ injection to the left breast. These results conform with what is reported by Losken et al. [4], who suggested that patients receiving greater volumes of fat injection show higher retention rates.

As seen in Figures 4 to 6 , the normalized difference in the Gaussian curvature between the pre and post fat graft images can be used to visualize changes in breast shape due to fat grafting. Differences in the surface curvature (white) can be seen in the fat graft area (delineated by the red outline) for the patients. The lower medial side of the breasts for Patients A and B appear to show the most changes in Gaussian curvature at the first time point after fat grafting, with a gradual reduction in the amount of change over time. Patient $C$ did not show much change in Gaussian curvature compared to the first two patients. It should be noted that this approach also highlights differences that can occur due to changes in posture, weight, and breathing as well as image misalignment. Unlike Gaussian curvature, the shape index and surface orientation images show changes throughout the fat graft region without any particular area of concentration.

\section{Patient A}

\section{Before fat graft 0 month}
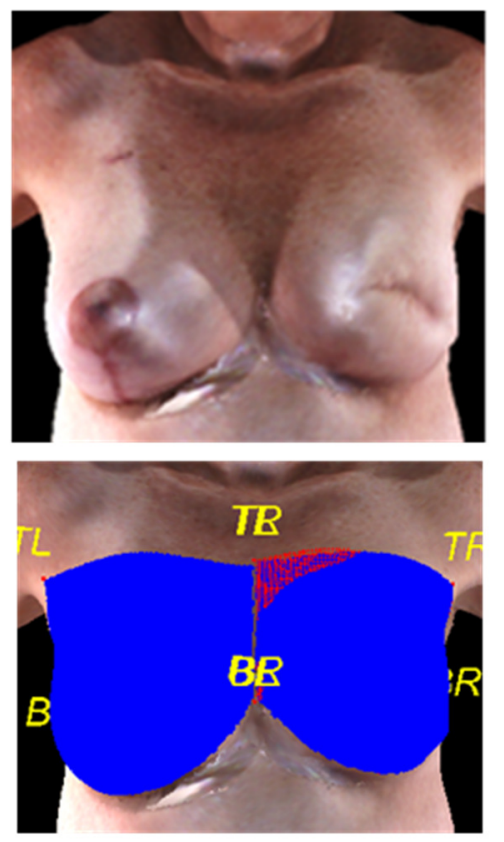

Right: 600 cc Left: 414 cc

\section{After fat graft 1 month}
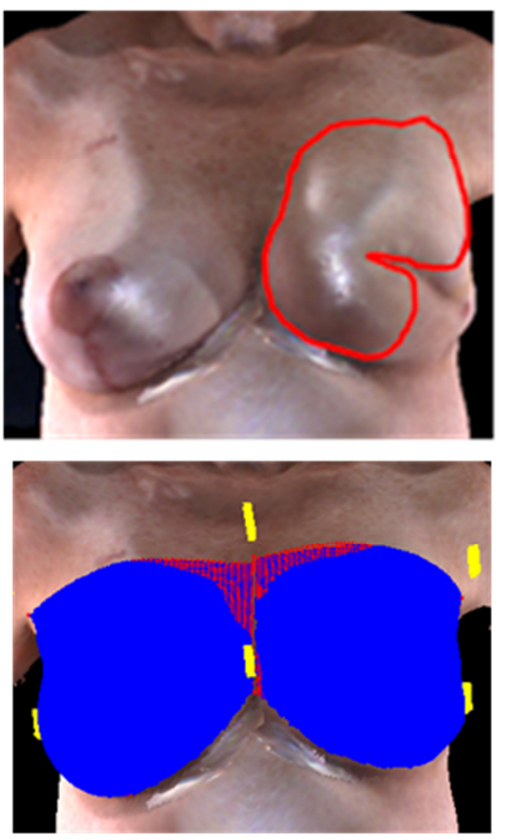

Right: 571 cc Left: 429 cc

\section{After fat graft 6 months}
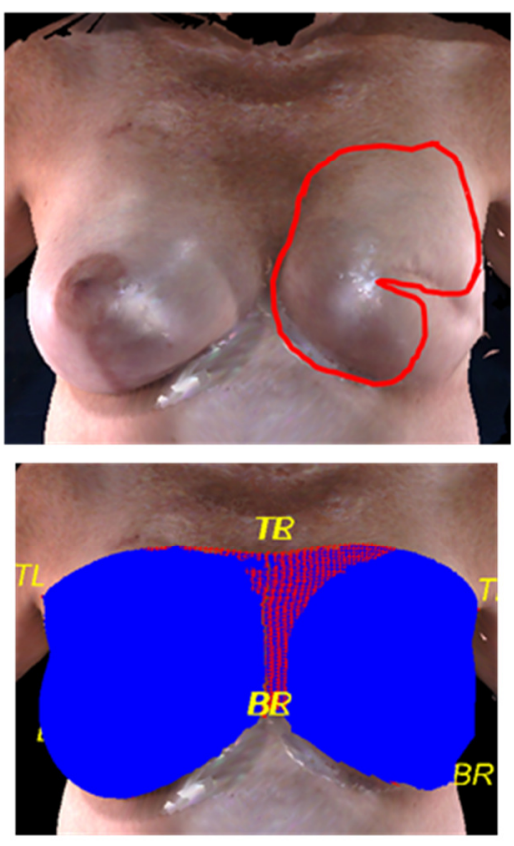

Right: 636 cc Left: $427 c c$

Fig. 1. Patient $A$, who previously underwent bilateral implant reconstruction, was imaged before fat grafting (pre-image) and after fat grafting (post-images). The red outlines demarcate the area of fat grafting in which $100 \mathrm{~mL}$ was injected. Volume was computed for the left and right breasts as shown by the second row of images and the measurement values below the images. 


\section{Patient B}

\section{Before fat graft 0 month}
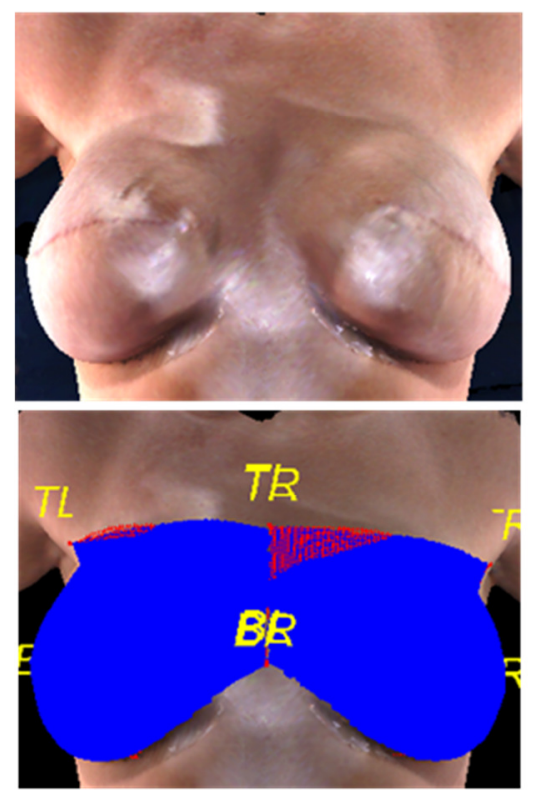

Right: 804 cc Left: $714 c c$

\section{After fat graft \\ 0.5 month}
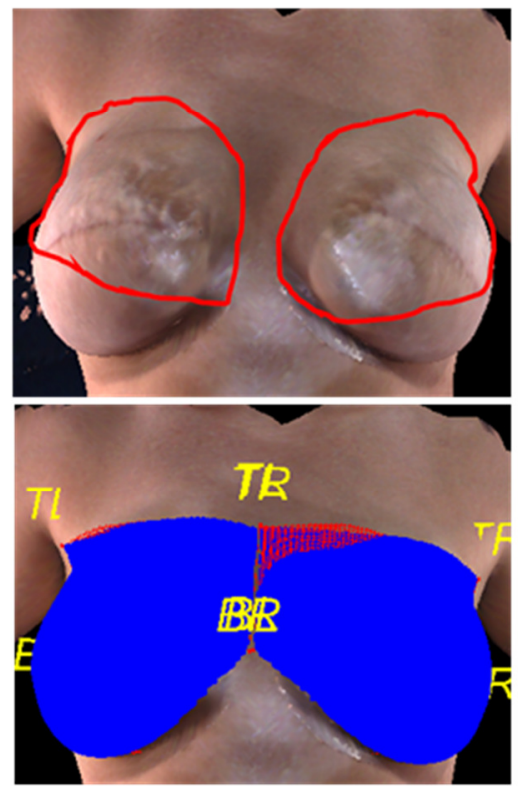

Right: 835 cc Left: 695 cc

\section{After fat graft \\ 3 months}

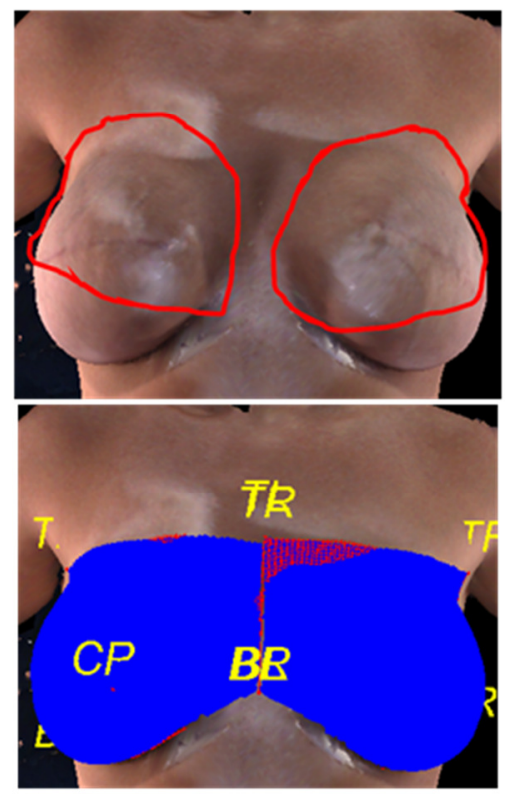

Right: $805 \mathrm{cc} \quad$ Left: $714 \mathrm{cc}$

Fig. 2. Patient $B$, who previously underwent bilateral implant reconstruction, was imaged before fat grafting (pre-image) and after fat grafting (post-images). The red outlines demarcate the area of fat grafting in which $60 \mathrm{~mL}$ was injected to each breast. Volume was computed for the left and right breasts as shown by the second row of images and the measurement values below the images.

\section{Patient C}

Before fat graft: 0 month
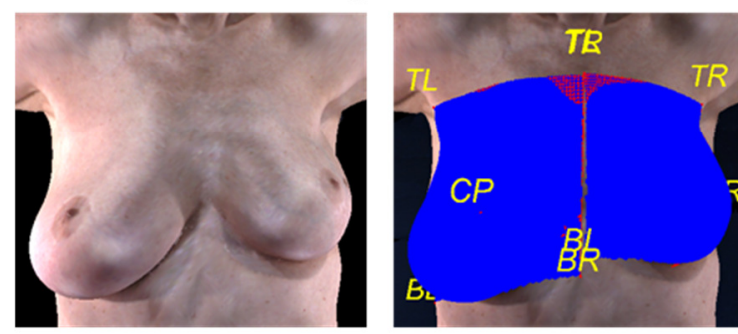

Right: 1089 cc Left: $563 c c$
After fat graft: 1 month
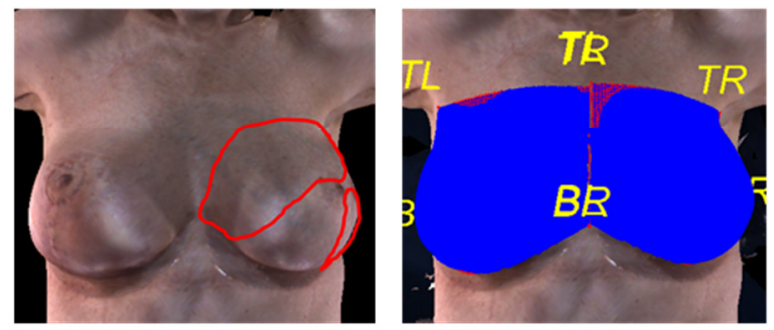

Right: $879 c c$ Left: $643 c c$

Fig. 3. Patient $C$, who previously underwent cosmetic bilateral mastopexy and then a lumpectomy, was imaged before fat grafting (pre-image) and after fat grafting (post-image). The red outlines demarcate the area of fat grafting. $240 \mathrm{cc}$ was injected to the upper breast outline and $120 \mathrm{cc}$ was injected to the lower lateral outline. Volume was computed for the left and right breasts with measurement values reported below the images. A revision was performed on the right breast for symmetry. 


\section{Patient A}

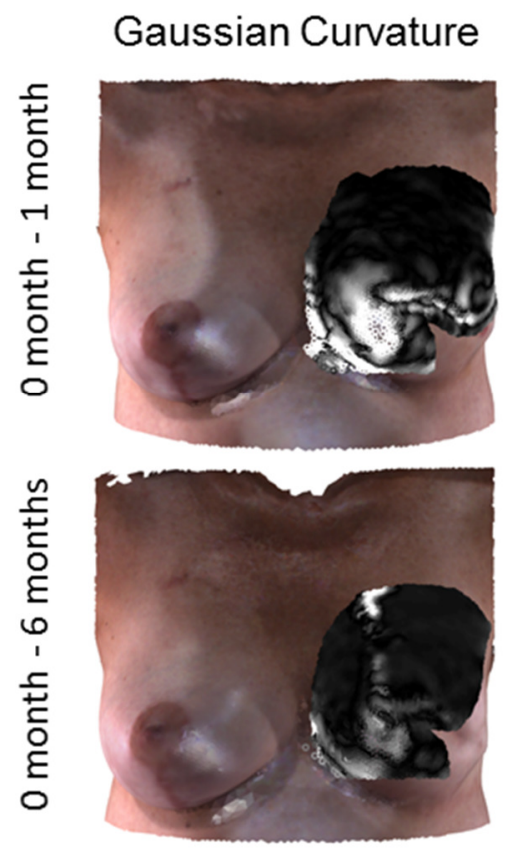

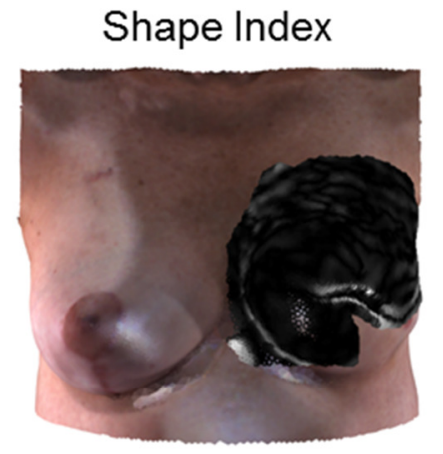

Surface Orientation

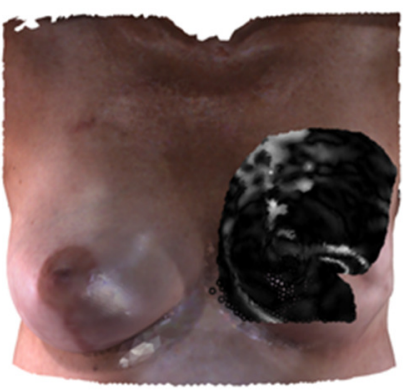

Fig. 4. Gaussian curvature, shape index, and surface orientation of the post-images (1 month and 6 months) were compared against the pre-image ( 0 month) of Patient $A$. White areas in the difference images represent high discrepancies between the pre- and post-images, whereas the gray shades represent smaller differences.

\section{Patient B}

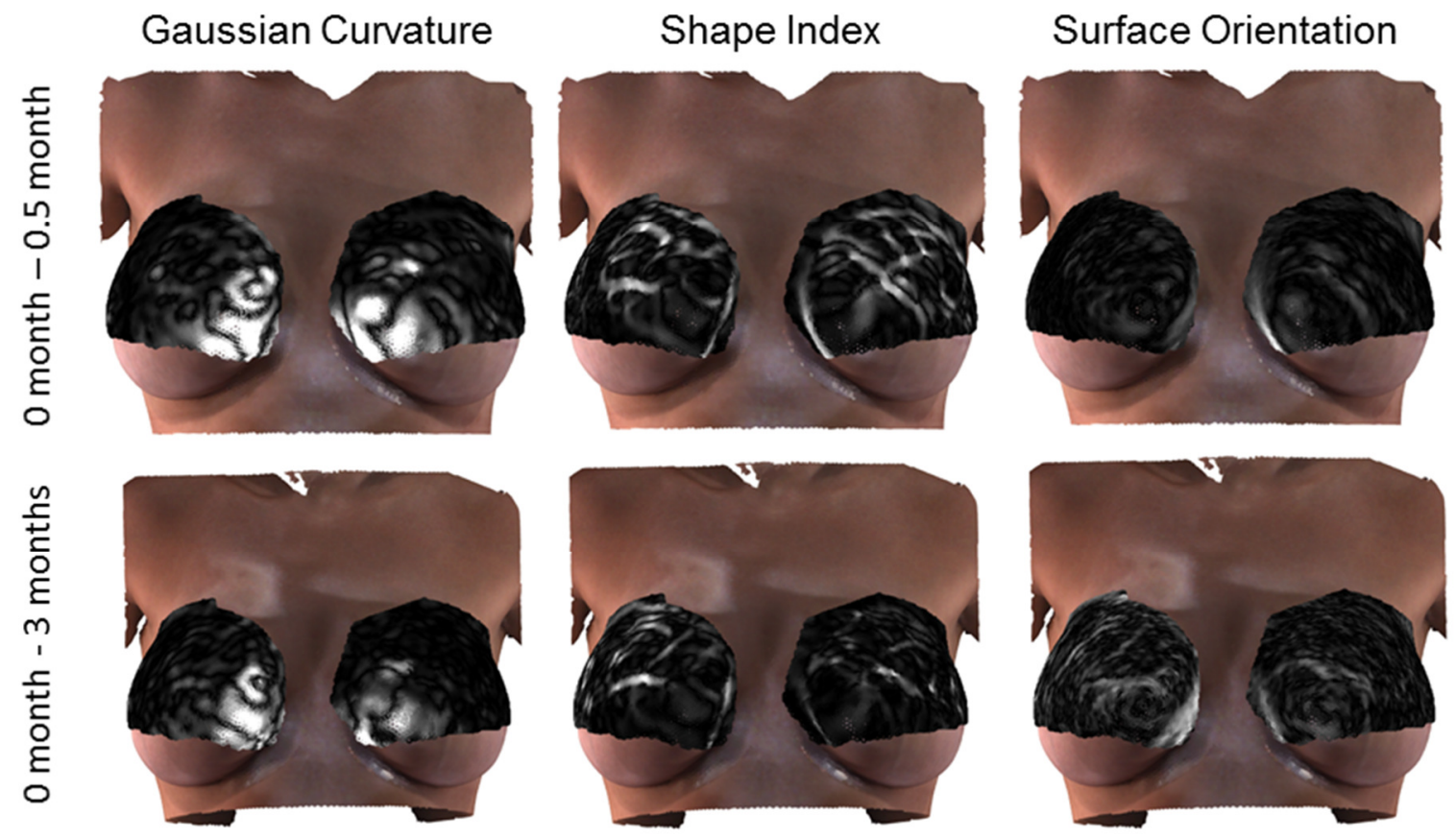

Fig. 5. Gaussian curvature, shape index, and surface orientation of the post-images ( 0.5 month and 3 months) were compared against the pre-image (0 month) of Patient $B$. White areas in the difference images represent high discrepancies between the pre- and post-images, whereas the gray shades represent smaller differences. 


\section{Patient C}

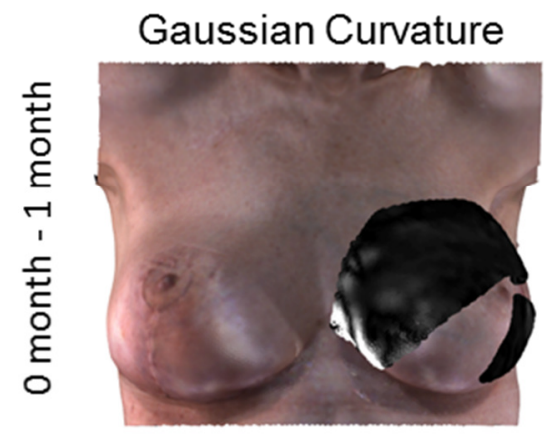

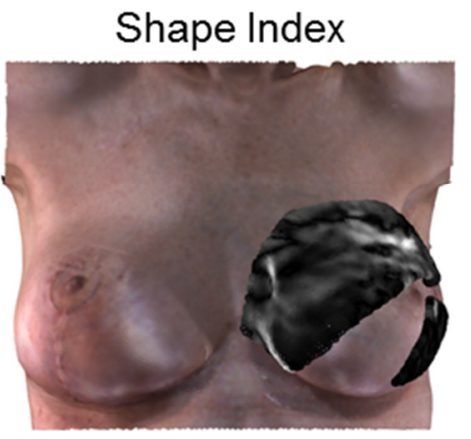

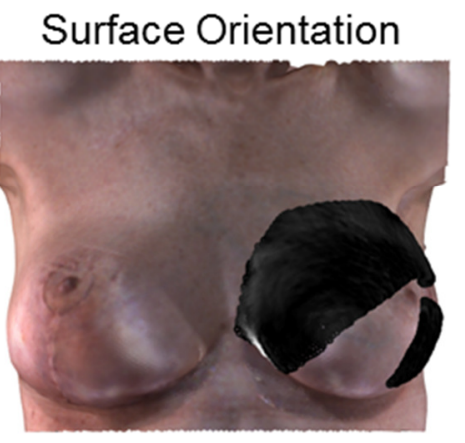

Fig. 6. Gaussian curvature, shape index, and surface orientation of the post-image (1 month) was compared against the pre-image ( 0 month) of Patient $C$. White areas in the difference images represent high discrepancies between the pre- and post-images, whereas the gray shades represent smaller differences.

\section{Conclusion}

The objective metrics demonstrated in this study using three-dimensional surface images were able to reveal visual changes in breast shape in the regions that were fat grafted. The breast volume was also measured to evaluate fat retention over time. Although changes were observed in the volume of the breasts before and after grafting, it should be noted that changes in volume less than $50 \mathrm{ml}$ are difficult to precisely quantify because small changes may also occur due to variations in landmark point selection, breathing, posture differences, weight changes, and other factors. As opposed to measuring the entire breast mound volume, future analysis can restrict volume measurements to the specific regions of the breast that are fat grafted to monitor changes in breast shape and rates of graft resorption. Further analysis will advance our understanding of breast shape and volume change following the procedure, improving the overall outcomes and expectations of fat grafting in breast reconstruction.

\section{Acknowledgements}

This material is based upon work supported by the National Institutes of Health under grant R01CA203984.

\section{References}

[1] P. Smith, W. P. Adams, A. H. Lipschitz, B. Chau, E. Sorokin, R. J. Rohrich, and S. A. Brown, "Autologous human fat grafting: Effect of harvesting and preparation techniques on adipocyte graft survival," Plast. Reconstr. Surg., vol. 117, no. 6, pp. 1836-1844, 2006.

[2] G. A. Ferraro, F. De Francesco, V. Tirino, C. Cataldo, F. Rossano, G. Nicoletti, and F. D'Andrea, "Effects of a new centrifugation method on adipose cell viability for autologous fat grafting," Aesthetic Plast. Surg., vol. 35, no. 3, pp. 341-348, 2011.

[3] I. Sarfati, R. F. D. van la Parra, C. A. Terem-Rapoport, D. Benyahi, C. Nos, and K. B. Clough, "A prospective randomized study comparing centrifugation and sedimentation for fat grafting in breast reconstruction," J. Plast. Reconstr. Aesthetic Surg., vol. 70, no. 9, pp. 1218-1228, 2017.

[4] M. Choi, K. Small, C. Levovitz, C. Lee, A. Fadl, and N. S. Karp, "The volumetric analysis of fat graft survival in breast reconstruction," Plast. Reconstr. Surg., vol. 131, no. 2, pp. 185-191, 2013.

[5] M. Herly, M. Ørholt, P. V. Glovinski, C. B. Pipper, H. Broholm, L. Poulsgaard, K. Fugleholm, C. Thomsen, and K. T. Drzewiecki, "Quantifying long-term retention of excised fat grafts: A longitudinal, retrospective cohort study of 108 patients followed for up to 8.4 years," Plast. Reconstr. Surg., vol. 139, no. 5, pp. 1223-1232, 2017.

[6] A. Losken, X. A. Pinell, K. Sikoro, M. V. Yezhelyev, E. Anderson, and G. W. Carlson, "Autologous fat grafting in secondary breast reconstruction," Ann. Plast. Surg., vol. 66, no. 5, pp. 518-522, 2011. 
[7] P. Gentile, C. Di Pasquali, I. Bocchini, M. Floris, T. Eleonora, V. Fiaschetti, R. Floris, and V. Cervelli, "Breast reconstruction with autologous fat graft mixed with platelet-rich plasma," Surg. Innov., vol. 20, no. 4, pp. 370-376, 2013.

[8] A. L. Cheong, G. P. Reece, M. K. Markey, M. C. Fingeret, and F. A. Merchant, "Quantitative Analysis of Localized Changes in Breast Shape," in Proceedings of 3DBODY.TECH 2017 - 8th International Conference and Exhibition on 3D Body Scanning and Processing Technologies, Montreal QC, Canada, 11-12 Oct. 2017, 2017, pp. 130-138.

[9] G. Peyre, "Toolbox graph.," 2013. [Online]. Available: http://www.mathworks.com/ matlabcentral/\%0Afileexchange/5355-toolbox-graph.

[10] D. Cohen-Steiner and J.-M. Morvan, "Restricted delaunay triangulations and normal cycle," Proc. Ninet. Conf. Comput. Geom. - SCG '03, p. 312, 2003.

[11] P. Alliez, D. Cohen-Steiner, O. Devillers, B. Lévy, and M. Desbrun, "Anisotropic polygonal remeshing," ACM Trans. Graph., vol. 22, no. 3, p. 485, 2003.

[12] G. P. Reece, F. Merchant, J. Andon, H. Khatam, K. Ravi-Chandar, J. Weston, M. C. Fingeret, C. Lane, K. Duncan, and M. K. Markey, "3D surface imaging of the human female torso in upright to supine positions," Med. Eng. Phys., vol. 37, no. 4, pp. 375-383, Apr. 2015. 